\title{
Chemical Factors in the Germination of Spore-bearing Aerobes: Observations on the Influence of Species, Strain and Conditions of Growth
}

\author{
By G. M. HILLS \\ Microbiological Research Department, Porton, near Salisbury, Wiltshire
}

SUMMARY: Some chemical requirements for stimulating the germination of spores of a few species of the genus Bacillus were studied by means of viable counts.

Of the three compounds alanine, tyrosine and adenosine, x-alanine was present in all cases where highly significant stimulation was observed; the need for the other two compounds ranged from ability to dispense completely with both of them to a distinct need for the two together to produce a maximum effect.

Generally, the effects were greater with spores grown in a chemically defined amino-acid medium aerated by shaking than with those grown on the surface of a casein-hydrolysate agar.

In the single case examined, that of a laboratory strain of $B$. subtilis, the effect of $\mathrm{L}$-alanine was strongly inhibited D-alanine at a molar ratio of $1: 30$.

Previous papers (Hills, 1949 $a, b$ ) have shown that the germination of spores of a virulent strain of Bacillus anthracis was stimulated by L-alanine, L-tyrosine and adenosine. Germination was strongly inhibited by D-alanine, and this inhibition was abolished by increasing the concentration of the L-isomer. After the discovery that only L-alanine was essential for rapid germination of the spores of a laboratory strain $B$. subtilis, a systematic examination of a number of species of the genus Bacillus was undertaken in order to see how far the chemical requirements for germination were common to the genus and how far they could be correlated with classification within the genus. The survey was not completed, but the data now available may be of interest to other workers studying the germination of spores.

\section{METHODS}

Strains used. Most of the work was done with a strain of B. subtilis isolated in this department. A virulent strain of B. anthracis supplied by $\operatorname{Dr} \mathbf{R}$. L. Vollum was that used previously by Hills $(1949 a, b)$. An avirulent strain of the same species, 34 F 2, was supplied by the Veterinary Laboratory of the Ministry of Agriculture, Weybridge. Other organisms were from the National Collection of Type Cultures (see Table 5).

Production of spore suspensions. Work with B. subtilis (laboratory strain) was carried out with a stock suspension which had been grown for $48 \mathrm{hr}$. on CCY agar (Gladstone \& Fildes, 1940), heated $90 \mathrm{~min}$. at $60^{\circ}$ at a count of about $10^{10}$ spores $/ \mathrm{ml}$. and washed thrice with distilled water at a count of $2 \times 10^{9} / \mathrm{ml}$.

For comparative work with other organisms, since it was considered that germination might be dependent on the method of cultivation, two different conditions of growth were used with each strain: $(a)$ surface growth on CCY agar; $(b)$ submerged growth, aerated by shaking, in the chemically defined 
medium (AA) of Gladstone (1939) but with L-alanine in place of DL-alanine (Hills, 1949b) and supplemented with $1.25 \times 10^{-6} \mathrm{M}$ adenosine (Hills, 1949a), $5 \times 10^{-4} \mathrm{M}-\mathrm{Ca}^{++}, 6 \times 10^{-3} \mathrm{M}^{+N_{2}} \mathrm{HCO}_{3}$ and $1.4 \times 10^{-5} \mathrm{M}-\mathrm{Mn}^{++}$(Brewer, McCullough, Mills, Roessler, Herbst \& Howe, 1946) and $10^{-7} \mathrm{M}$ aneurin (Hagan, O'Kane \& Young, 1943). The metals were held in solution by $\mathrm{m} / 100$ citrate.

For optimal sporulation of some strains at $37^{\circ}$, growth on medium $(a)$ was continued for 12 days. The organisms were then suspended in glass-distilled water, collected by centrifuging and washed. In medium $(b)$ optimum sporulation was observed at 5 days. The organisms were then centrifuged out and washed. These times for optimum production of free spores were determined by microscopic examination, but viable counts of both types of suspension showed that the proportion of vegetative forms sensitive to heat $\left(15 \mathrm{~min}\right.$. at $\left.60^{\circ}\right)$ was insignificant. These suspensions were used without heating to avoid the possibility, frequently reported (e.g. Robbins, Kavanagh \& Kavanagh, 1942; Evans \& Curran, 1943) of facilitating germination by heat treatment.

Experimental procedure. The media in which germination was studied were inoculated with about $10^{4} \mathrm{spores} / \mathrm{ml}$. and incubated at $35^{\circ}$. Counts of organisms which then remained viable after $15 \mathrm{~min}$. at $60^{\circ}$ (spore, or $S$-counts) were determined by the surface plate technique of Miles $\&$ Misra (1938). In selected experiments counts made without heating at $60^{\circ}(S+V$ counts, where $V$ is the count of the vegetative form) showed little fall as a result of incubation, but in suitable media comparison with the $S$-counts showed that a high proportion of the spores had become sensitive to heat. Since these had remained viable during incubation they must be regarded as having germinated. In certain media, however, which were inadequate for growth though adequate for the process of sensitization to heat, loss of viability of the heat-sensitive forms was observed even at $35^{\circ}$ on prolonged incubation. Nevertheless, in spite of difficulties due to death of germinated forms in media inadequate for growth, increased sensitivity to heat, as determined by viable count, is the best criterion of germination, since any method based solely on morphological changes cannot establish the most important feature of the process, namely, that changes from the characteristics of the spore state have not involved loss of viability. Further evidence in support of this view has recently been given by Wynne \& Foster (1948). Technical details have been given elsewhere (Hills, 1949a), the only modifications being in the conditions of incubation of the plates according to the needs of the various organisms for the production of discrete colonies, uniformly 1-2 mm. in diameter.

Statistical treatment of data. The numbers of colonies on replicate plates conformed to a Poisson series, so that in assessing the significance of the differences between counts, according to standard methods (Fisher, 1946), the variance of any count could be taken equal to the count itself.

Materials. These were as used previously (Hills, 1949a, b). Throughout, molarities refer to a single enantiomorph; the total concentration of a DLmixture is thus twice that given. 


\section{RESULTS}

\section{Experiments with Bacillus subtilis}

Selection of medium. Fig. 1 shows the changes in $S$-count in a variety of media during $6 \mathrm{hr}$. incubation. These media fell into three groups:

(a) Disappearance of $50 \%$ or more spores in the first $2 \mathrm{hr}$. occurred only in the amino-acid medium (A), in CCY, and in media containing tryptic digest of meat (TM and TM + PB). The initial fall in the number of spores with CCY occurred $1 \mathrm{hr}$. later than in the other media, but the subsequent decrease in $S$-count was rapid, followed later by an increased count due to resporulation.

(b) In the second group, which gave significant but less than $50 \%$ germination in the first $2 \mathrm{hr}$. and little further increase, the gelatin-tyrosine medium (GT), developed for the assay of yeast fractions in the germination of $B$. anthracis, was not significantly different from phosphate buffer (B), and was not improved by yeast extract (GTY).

(c) The replacement of the amino-acids of medium (A) by ammonium salts inhibited germination since the medium $\left(\mathrm{NH}_{4}\right)$ allowed less change in spore count than the phosphate buffer (B) which it contained.

All the changes observed were presumably due to germination, since considerable growth occurred in those cases with the most rapid decrease in $S$-count. This was shown by very high $S+V$ counts at the end of the experiment, using the same sized sample as for the $S$-counts but without heating at $60^{\circ}$. Some growth also occurred in the gelatine-tyrosine medium with yeast (GTY), giving a twofold increase in the count of an unheated dilution, but in the remaining three media, $\mathrm{B}, \mathrm{GT}$ and $\mathrm{NH}_{4}$, this count was either lower than or not significantly different from the initial count.

Table 1. Effect of incubation in amino-acid media on spores of a strain of Bacillus subtilis

All media were buffered with $\mathrm{M} / 30$ phosphate, $\mathrm{pH} 7 \cdot 8$. $S=$ spore count; $S+V=$ spore and vegetative form count.

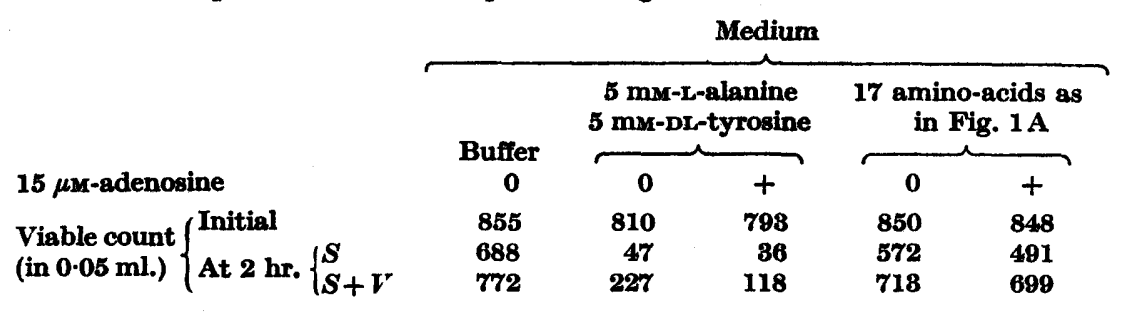

Since measurable rates of germination were observed in the 'complete' amino-acid medium, it was compared with a simpler amino-acid medium (Table 1) containing L-alanine and tyrosine which, with the addition of adenosine, had been found adequate for $B$. anthracis. Fig. 1 shows that even with the 'complete' medium there was only 50 and $70 \%$ germination in 1 and $2 \mathrm{hr}$. respectively; the latter experimental period therefore was chosen. The simpler medium, however, caused a much more rapid fall in $S$-count, but in 


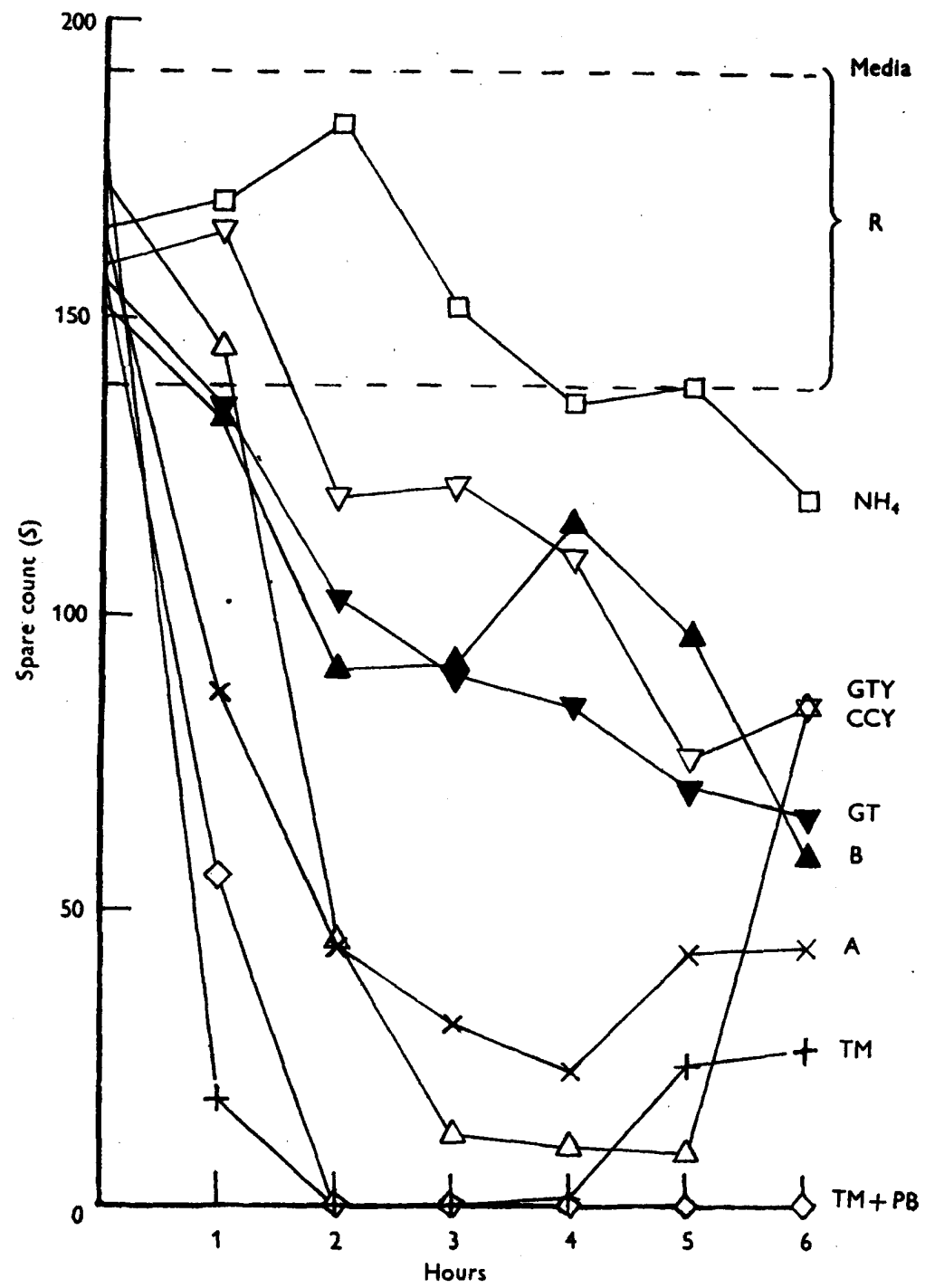

Fig. 1. The effect of incubation $\left(\mathbf{8 5}^{\circ}\right)$ in various media on $S$-counts with a strain of $B$. subtilis. The sample for each count was $0.2 \mathrm{ml}$. of a $1 / 10$ dilution in $\mathrm{M} / 80$-phosphate buffer pH $7 \cdot 8$. The count of spores and vegetative cells together $(S+V)$ at the end of the experiment is indicated with the key to the media used. $R=$ range of counts not signiflcantly different from the mean initial count $(P=0.05)$.

Media (with final $S+V$ count): A, 17 amino-acids (Hills, 1949b, Table 1, with Na citrate but no threonine), $\times(\infty) ; \mathrm{B}, \mathrm{M} / \mathbf{3 0}$ phosphate buffer $\mathrm{pH} \mathrm{7 \cdot 3,} \Delta$ (105); CCY, acid + tryptic hydrolysates of casein + yeast extract (Gladstone \& Fildes, 1940), $\Delta(\infty)$; GT, gelatin hydrolysate + tyrosine (Hills, 1949a), $\nabla$ (162); GTY, GT + yeast extract, $\nabla$ (861); $\mathrm{NH}_{4}$, ammonium salts at concentrations used by Fildes (1988) in place of amino-acids of A, $\square$ (140); TM, tryptic digest of beef $+(\infty)$; TM + PB, TM + Fildes's peptic sheep's blood $\bigcirc(\infty)$. 
$2 \mathrm{hr}$. the $S+V$ count also fell considerably, so that it was not possible to ascribe the decreased $S$-count to germination, rather than to a sporicidal effect. The only significant effect of adenosine in this medium was a greater decrease in $S+V$ count. In a subsequent experiment, limited to $1 \mathrm{hr}$. incubation (Table 2), L-alanine was the only component essential for the decrease in

Table 2. Effect of alanine and tyrosine on germination of a strain of Bacillus subtilis

The basal medium was $\mathrm{M} / 30$ phosphate $\mathrm{pH} 7 \cdot 3$, and the amino-acids were added at a concentration $5 \mathrm{~mm}$.

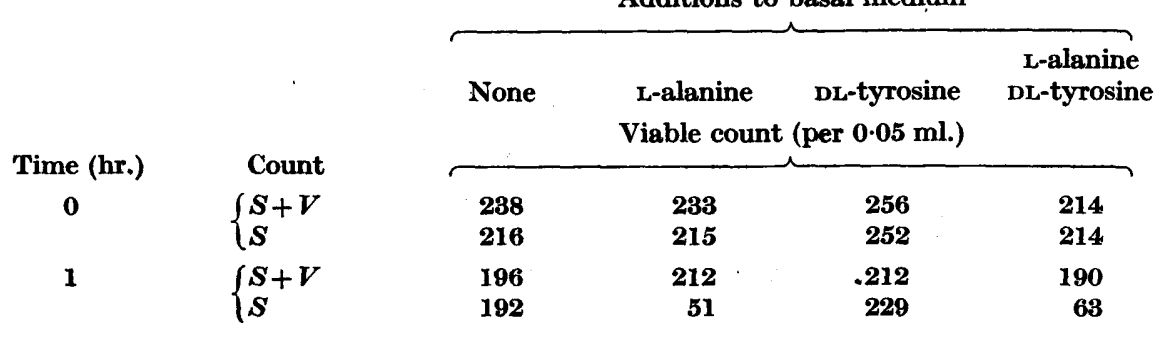

$S$-count, but it had no effect on the total number of viable organisms, which, although showing a 10-20\% decrease as a result of incubation, showed the same decrease when in phosphate buffer alone. Since $75 \%$ germination was observed in $1 \mathrm{hr}$., the experimental period was shortened to $30 \mathrm{~min}$. in subsequent experiments and good germination still took place.

Alanine was not replaceable by lactate or pyruvate, even at 5-10 times the alanine concentration and in the presence of an equivalent of $\mathrm{NH}_{4}{ }^{+}$, though none of these compounds significantly inhibited the decrease in $S$-count caused by alanine. Thus in $28 \mathrm{~mm}$. phosphate buffer of $\mathrm{pH} \mathrm{7 \cdot 3}$, buffer with $5 \mathrm{mM}-\mathrm{NH}_{4} \mathrm{Cl}$, $5 \mathrm{~mm}$ pyruvate, pyruvate $+\mathrm{NH}_{4} \mathrm{Cl}, 2 \cdot 5 \mathrm{~mm}$-DL-lactate, DL-lactate $+\mathrm{NH}_{4} \mathrm{Cl}$, the count, initially in the range 669-717, decreased slightly to 627-662 after incubation for $30 \mathrm{~min}$. With $\mathbf{0 . 5} \mathrm{mm}$-L-alanine in addition to any of the above substrates or combinations of substrates the final count was in the range 123-162.

Inhibition by D-alanine. As with $B$. anthracis the decrease in $S$-count caused by L-alanine was inhibited by D-alanine, a molecular ratio of $0.03 \mathrm{D}$ - to $\mathrm{L}$-alanine allowing about twice as many spores to survive as with L-alanine alone at 0.5-5 mM (Table 3). Controls with the brucine and strychnine used in the resolution of DL-alanine showed a small inhibition of germination at $20 \mu \mathrm{g} . / \mathrm{ml}$. in the presence of $500 \mu \mathrm{M}$-L-alanine, but even $1.35 \mu \mathrm{g} . / \mathrm{ml}$. D-alanine $(15 \mu \mathrm{M})$ caused an inhibition about three times as great. This effect of D-alanine therefore cannot be due to traces of these alkaloids as impurities.

\section{Experiments with other organisms}

Table 4 shows the effects on the $S$-counts after incubation in phosphate buffer $\mathrm{pH} 7 \cdot 3$ with the addition of L-alanine, DL-tyrosine and adenosine singly and in all possible combinations. The organisms used were four strains of 
$B$. anthracis and a strain each of $B$, cereus, B. megatherium and B. subtilis. Two spore suspensions of each organism were used, grown respectively on the surface of CCY agar (Gladstone \& Fildes, 1940) and in an amino-acid fluid medium aerated by shaking (see Methods).

Table 3. Inhibition of germination of a strain of Bacillus subtilis by D-alanine

The basal medium was $\mathrm{M} / 30$-phosphate, pH 7·3. The initial $S$-counts, both with and without $D-$ or L-alanine, formed a homogeneous population of range 515-611 $(P=0.95)$.

Conc. of D-alanine $(\mu \mathrm{M})$

\begin{tabular}{|c|c|c|c|c|}
\hline $\begin{array}{c}\text { Conc. of } \\
\text { L-alanine }\end{array}$ & \multicolumn{4}{|c|}{$S$-count at $30 \mathrm{~min}$. } \\
\hline 0 & 529 & 528 & 612 & 622 \\
\hline 500 & 157 & 341 & $\mathbf{4 6 7}$ & 594 \\
\hline 1500 & 129 & 160 & 235 & 388 \\
\hline 5000 & 79 & 85 & 118 & 162 \\
\hline
\end{tabular}

No experiments were carried out with either $\boldsymbol{B}$. mesentericus or $\boldsymbol{B}$. mycoides. Two strains of the former (N.C.T.C. nos. 2589 and 6223) although sporing satisfactorily on CCY agar, failed to grow in the defined medium; and two strains of mycoides (N.C.T.C. nos. 926 and 2602) did not spore satisfactorily on either medium and were also unsuitable for study by the technique used, on account of the difficulty of getting discrete colonies for counting.

Except with $B$. cereus those conditions which produced a significant decrease in $S$-count gave a greater decrease with spores grown in the amino-acid medium than with those grown on CCY. Although this difference was, in general, quantitative rather than qualitative, it led in certain cases to qualitative differences due to limitations of the counting technique in detecting small changes (5-18\% depending on the total number of colonies for both counts of which the significance of the difference was required). Thus with $B$. anthracis, N.C.T.C. 5444, $79 \%$ of spores grown on the amino-acid medium were susceptible to the action of alanine and tyrosine together but the corresponding figure of $15 \%$ for spores of this strain grown on CCY agar was not significantly different from the control. On the other hand, with B. anthracis, N.C.T.C. 109, both figures, 88 and $25 \%$ respectively, were significant.

The simplest requirements for spore germination were shown by $\boldsymbol{B}$. cereus for which alanine alone was effective, though there was some improvement with tyrosine or adenosine, and these two together, without alanine, gave a small but significant effect. All four strains of $B$. anthracis, whether virulent or avirulent, had similar requirements. Alanine, tyrosine and adenosine together were significantly better than any single component or combination of two components. Again, alanine seemed to be of greatest importance since only two cases in 24 showed a significant effect $(P=0 \cdot 95)$ in its absence, and in one of these cases the decrease in $S$-count was so small that the probability of such a difference in the same direction arising by chance was nearly 1 in 50 . With both the Weybridge and Vollum strains, the effect of alanine in diminishing 


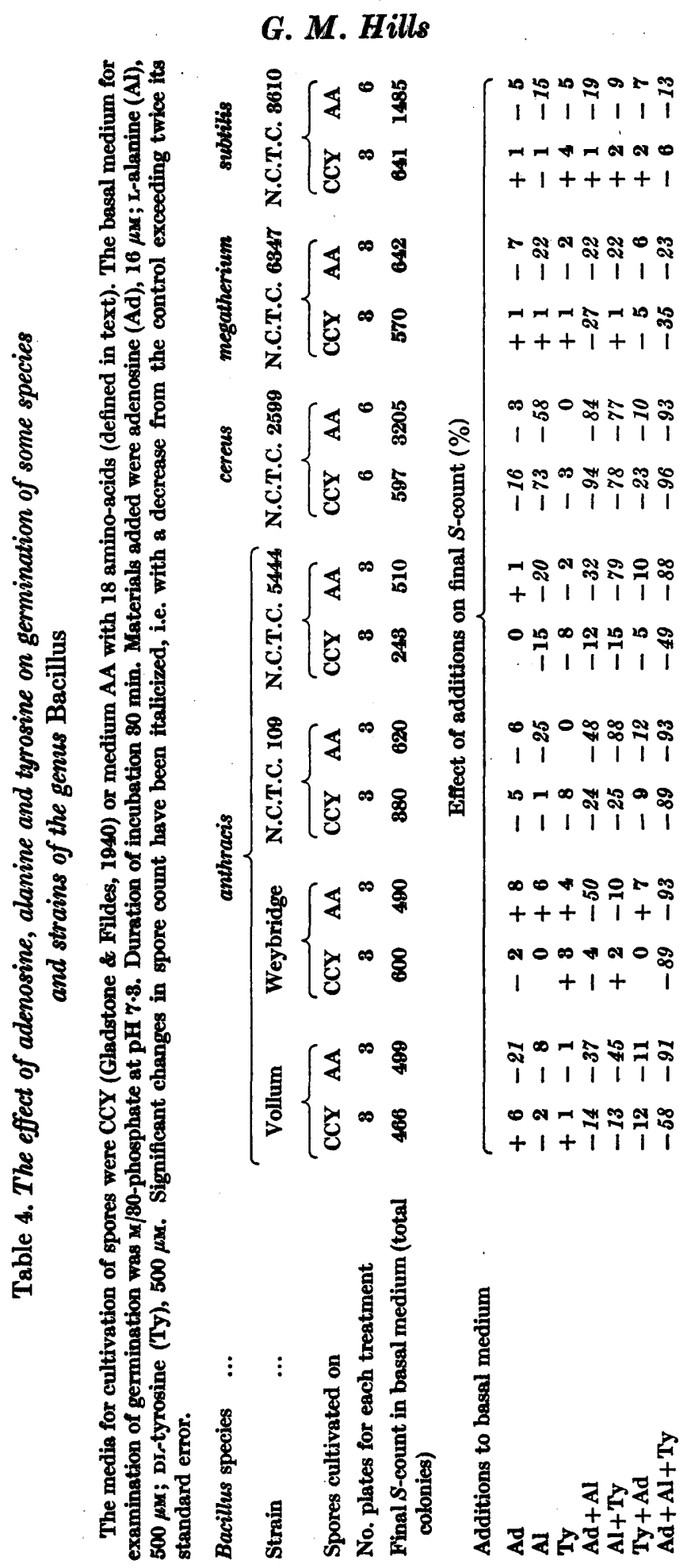




\section{Chemical factors in aerobic spore germination}

the $S$-count in the presence of tyrosine and adenosine was subsequently shown to be due to germination. Under conditions in which the $S$-count fell to $10-35 \%$ of its original value in the presence of alanine, the $S+V$ count in the presence or absence of alanine and the $S$-count in the absence of alanine fell only to $85-95 \%$ of the original value of the $S+V$ count, of which the original $S$-count was 95-99\% $(P=0 \cdot 95)$. Germination of $B$. megatherium and $B$. subtilis was slower so that large decreases in $S$-count were not observed during the period of the experiment. Significant decreases were observed only in the presence of alanine, but these were small and were not affected, in the case of cultures grown in the amino-acid medium, by the other components either singly or together. During the period of the experiment, $S$-counts gave no evidence for an effect of any of the materials on the germination in phosphate buffer of $B$. subtilis spores grown on the surface of CCY agar. A significant fall in the count of spores of $B$. megatherium, grown on CCY agar, was produced only in the presence of alanine and adenosine together, with or without tyrosine.

\section{DISCUSSION}

This work was begun in order to seek a correlation between chemical requirements and mode of germination, such as the differences observed by Lamanna (1940), namely, absorption of the spore-coat in large-celled species and shedding of the spore-coat in small-celled species. No generalization is possible since too few species have been studied, and strain variations in $B$. anthracis suggest the need for the study of a number of strains of each species. The variations obtained with the strains of $\boldsymbol{B}$. anthracis, however, were quantitative rather than qualitative. All strains were more exacting in the need for tyrosine and adenosine than the single strain of $B$. cereus studied. The smaller variations in requirements within the species were not correlated with virulence; thus the virulent 'Vollum' was less exacting than the avirulent 'Weybridge', but more so than the avirulent N.C.T.C. no. 54,44.

The failure of B. mycoides (N.C.T.C. nos. 926 and 2602) to spore under the conditions used was unfortunate, since the work of Knaysi (1945) indicates requirements for germination in this species different from those of the organisms studied here. Using a microscopic technique, Knaysi found that a nitrogen source was unnecessary, buffered glucose being adequate for $30 \%$ germination in $\mathbf{5 ~ h r}$.; $\mathrm{KNO}_{3}$ facilitated the conversion of germinating forms to true vegetative cells. His conditions, however, must have been far from optimal, since Robinow (1942), studying cytological changes during germination, has shown that in broth marked changes occur during the first 5 or $10 \mathrm{~min}$. of incubation and that cell division occurred in about $1 \mathrm{hr}$. These rates are comparable with those observed with the organisms studied here. Moreover, Knaysi \& Baker (1947) have explained germination in nitrogen-free media by exhaustion of cellular ribonucleic acid, so that some nitrogen source is essential to give normal, as distinct from deficient vegetative cells.

Owing to the use of different strains and experimental conditions, the results with $B$. subtilis are not directly comparable with those of Keilin \& Hartree 
(1947), who found that N.C.T.C. no. 89 needed a stable dialysable factor from yeast extract, peptone or tryptic digest of casein. The present findings are that L-alanine in phosphate buffer was a sufficient stimulus for a laboratory strain, but N.C.T.C. no. 3610 did not respond, in a short incubation period, either to this or to the alanine + tyrosine + adenosine medium which was adequate for $B$. anthracis, and in which adenosine replaced a yeast extract (Hills, 1949a) having similar properties to that used by Keilin \& Hartree.

The changes in $S$-count have not in every case been proved to be due to germination, but this was the case with two strains of $B$. anthracis and with a laboratory strain of $\boldsymbol{B}$. subtilis. With $\boldsymbol{B}$. subtilis loss of viability was observed in the absence of heat treatment only after prolonged incubation of suspensions containing germinated forms in media inadequate for growth. It is probable, therefore, that germination was the cause of the decrease in $S$-count in experiments of short duration with other species.

L-alanine was a constituent of the medium in 40 cases out of 42 where the decrease in $S$-count exceeded that which would have been expected by chance in 1 case in 50. The importance of L-alanine is emphasized by the inhibition of its effect by D-alanine at a molar ratio of $1: 30$ in the case of $B$. anthracis (Hills, $1949 b$ ) and by a laboratory strain of $B$. subtilis which required only L-alanine to stimulate $80 \%$ germination in $1 \mathrm{hr}$. at $35^{\circ}$. The specificity of the effects of the isomers of alanine on the germination of bacterial spores is at present unique in bacterial chemistry in the low ratio to L-alanine at which $\mathrm{D}$-alanine inhibits. The inhibition of Lactobacillus arabinosus by $\mathrm{D}$-leucine and D-valine (Fling \& Fox, 1945) or by glycine (Kobayashi, Fling \& Fox, 1948) and the inhibition of Escherichia coli by these amino-acids or by D-alanine (Kobayashi, Fling \& Fox, 1948) required high concentrations approaching $0 \cdot 1 \mathrm{M}$. The view that the large side-chain of valine or leucine is essential for steric hindrance cannot now be regarded of general biological application, and its bearing on the mode of action of peptide antibiotics containing D-amino-acids (Fox, Fling \& Bollenbach, 1944) is now in doubt (cf. Work, 1948).

I have much pleasure in thanking Dr D. W. Henderson and Dr D. D. Woods for careful criticism of this paper and Dr H. N. Rydon for samples of D- and L-alanine and synthetic DL-tyrosine. The experimental work was carried out with the technical assistance of Cpl. W. Bailey, R.A.M.C., while the author was a member of the Scientific Staff, Medical Research Council. Permission to publish has been granted by the Chief Scientist, Ministry of Supply.

\section{REFERENCES}

Brewer, C. R., McCullough, W. G., Milus, R. C., Roessler, W. G., Herbst, E. J. \& Howe, A. F. (1946). Studies on the nutritional requirements of Bacillus anthracis. Arch. Biochem. 10, 65.

Evans, F. R. \& Curran, H. R. (1943). The accelerating effect of sub-lethal heat on spore germination in mesophilic aerobic bacteria. J. Bact. 46, 513 .

Fildes, P. (1938). The growth of Proteus on ammonium lactate plus nicotinic acid. Brit. J. exp. Path. 19, 239.

Fisher, R. A. (1946). Statistical methods for research workers, 10th ed. Edinburgh: Oliver and Boyd. 
Fung, M. \& Fox, S. W. (1945). Antipodal specificity in the inhibition of Lactobacillus arabinosus by amino-acids. J. biol. Chem. 160, 329.

Fox, S. W., Fling, M. \& Bollentach, G. N. (1944). Inhibition of bacterial growth by D-leucine. J. biol. Chem. 155, 465 .

Gladstone, G. P. (1939). ' Interrelationships between amino-acids in the nutrition of B. anthracis. Brit. J. exp. Path. $20,189$.

Gladstone, G. P. \& Fildes, P. (1940). A simple culture medium for general use without meat extract or peptone. Brit. J. exp. Path. 21, 161.

Hagan, W. A., O'Kane, D. J. \& Young, G. A. (1943). Private communication.

HrLls, G. M. (1949a). Chemical factors in the germination of spore-bearing aerobes. The effect of yeast extract on the germination of Bacillus anthracis and its replacement by adenosine. Biochem. J. 45, 353.

Hills, G. M. (1949b). Chemical factors in the germination of spore-bearing aerobes. The effect of amino-acids in the germination of Bacillus anthracis including observations on the specificity of some stereoisomers. Biochem. J. 45, 363 .

Keimin, D. \& Hartree, F. (1947). Comparative study of the spores and vegetative forms of Bacillus subtilis. Antonie van Leeurvenhoek J. Microbiol. Serol. 12, 115.

KNAYSI, G. (1945). Investigation of the existence and nature of reserve material in the endospore of a strain of Bacillus mycoides by an indirect method. J. Bact. 49, 617 .

KNaYsi, G. \& BaKer, R. F. (1947). Demonstration, with the electron microscope, of a nucleus in Bacillus mycoides grown in a nitrogen-free medium. J. Bact. $53,539$.

Kobayashi, Y., Fuing, M. \& Fox, S. W. (1948). Antipodal specificity in the inhibition of growth of Escherichia coli by amino-acids. J. biol. Chem. 174, 391.

Lamanna, C. (1940). The taxonomy of the genus Bacillus. 1. Modes of spore germination. J. Bact. 40, 347.

Mrles, A. A. \& Misra, S. S. (1938). The estimation of the bactericidal power of blood. J. Hyg., Camb., 38, 732.

Robrins, W. J., Kavanagh, V. W. \& Kavanagh, F. (1942). Growth substances and dormancy of spores of Phycomyces. Bot. Gaz. 104, 224.

Rosinow, C. F. (1942). A study of the nuclear apparatus of bacteria. Proc. Roy. Soc. B, 130, 299.

Work, T. S. (1948). D- and L-amino-acids in antibiotics. Biochem. Soc. Symp. 1, 61.

WYNNE, E. S. \& Foster, J. W. (1948). Physiological studies in spore germination with special reference to Clostridium botulinum. 1. Development of a quantitative method. J. Bact. 55, 61. 